\title{
Rocaglamide breaks TRAIL resistance in HTLV-1-associated adult T-cell leukemia/lymphoma by translational suppression of c-FLIP expression
}

\author{
M Bleumink ${ }^{1}$, R Köhler ${ }^{1}$, M Giaisi $^{1}$, P Proksch ${ }^{2}$, PH Krammer ${ }^{1}$ and M Li-Weber ${ }^{\star, 1}$
}

The human T-cell leukemia virus type-1 (HTLV-1)-associated adult T-cell leukemia/lymphoma (ATL) is incurable by currently known therapies. ATL samples and cell lines derived from ATL patients show restricted sensitivity to tumor necrosis factorrelated apoptosis-inducing ligand (TRAIL) and CD95 ligand (CD95L). We have recently shown that HTLV-1-infected cells express elevated levels of cellular caspase-8 FLICE-inhibitory protein (c-FLIP) conferring resistance to receptor-mediated apoptosis. This finding underscores the demand to develop new strategies for treatment of ATL. In this study, we show that the naturally occurring herbal compound Rocaglamide (Roc) sensitizes CD95L- and TRAIL-induced apoptosis in HTLV-1-infected cells by downregulation of c-FLIP expression. Investigation of the molecular mechanism of Roc-mediated downregulation of c-FLIP revealed that it inhibits phosphorylation of the translation initiation factor $4 \mathrm{E}$ (elF4E), a key factor that controls the rate-limiting step of translation, through inhibition of the MEK-ERK-MNK1 signaling pathway. This event prevents de novo synthesis of short-lived proteins such as c-FLIP in HTLV-1-infected cells. Our data suggest that Roc may serve as an adjuvant for TRAIL-based anticancer therapy.

Cell Death and Differentiation (2011) 18, 362-370; doi:10.1038/cdd.2010.99; published online 13 August 2010

Induction of apoptosis in tumor cells is one of the therapeutic strategies against different types of cancer. ${ }^{1-3}$ Apoptosis may be induced through the mitochondrial (intrinsic) pathway or through death receptors of the tumor necrosis factor (TNF) receptor superfamily, such as CD95 (APO-1/Fas) and the TNF-related apoptosis-inducing ligand (TRAIL) receptors, TRAIL-R1 and TRAIL-R2. ${ }^{3,4}$ Intrinsic death stimuli (e.g., anticancer drugs) directly or indirectly activate the mitochondrial pathway resulting in the release of cytochrome $c$ and sequential activation of caspases- 9 and 3, finally leading to apoptosis. ${ }^{2}$ The receptor-mediated apoptosis pathway is initiated by ligation of death receptors with their cognate ligands, such as TRAIL and CD95 ligand (CD95L), resulting in activation of caspases- 8 and 10 , which induces apoptosis by subsequent activation of the executioner caspase-3 or indirectly through the mitochondria by cleavage of the $\mathrm{Bcl}-2$ family protein Bid, which induces cytochrome $c$ release. ${ }^{3,4}$ One major negative regulator of receptor-mediated apoptosis is the cellular caspase-8 (FLICE)-inhibitory protein (c-FLIP), which blocks processing and activation of caspase-8 at the level of the death-inducing signaling complex. ${ }^{4}$

TRAIL, in contrast to the CD95L, shows virtually no toxicity on normal cells and, therefore, is a promising anticancer agent that is currently under clinical trails. ${ }^{3,5,6}$ Unfortunately, many cancer cells remain partially or completely resistant to
TRAIL, and in some resistant cancers, TRAIL even stimulates survival and proliferation. ${ }^{7,8}$ The human T-cell leukemia virus type-1 (HTLV-1)-associated adult T-cell leukemia/lymphoma (ATL) is a malignancy of clonally proliferating infected mature $\mathrm{CD}^{+}{ }^{+} \mathrm{T}$ cells. ${ }^{9}$ We and others have shown that primary HTLV-1-ATL samples and ATL cell lines derived from HTLV1 -infected patients are more resistant to TRAIL- and CD95Lmediated apoptosis compared with non-HTLV-1-infected leukemic cells. ${ }^{10-12}$ Worldwide HTLV-1 has infected $15-20$ million people. Patients have a poor prognosis with a survival range of less then 1 year after disease onset. So far, HTLV-1associated ATL is incurable by presently known therapies. ${ }^{9}$ Expression of the virus transactivator protein Tax has been found to correlate with resistance to TRAIL and CD95L. ${ }^{10,12}$ Recently, we and others have shown that HTLV-1 Tax increases c-FLIP expression, which is responsible for the resistance to receptor-mediated apoptosis. ${ }^{12,13}$ Downregulation of c-FLIP by SiRNA has been shown to be sufficient to sensitize various types of cancer cells, including the HTLV-1associated ATL to death ligands. ${ }^{12,13}$ Therefore, c-FLIP represents a promising therapeutic target, especially for TRAIL-based therapies.

c-FLIP expression is controlled at multiple levels. At the transcriptional level, c-FLIP is the target of the inducible transcription factors NF- $\kappa$ B and NF-AT. ${ }^{14,15}$ In addition, the

\footnotetext{
${ }^{1}$ Tumor Immunology Program (D030), German Cancer Research Center (DKFZ), Heidelberg, Germany and ${ }^{2}$ Institute of Pharmaceutical Biology and Biotechnology, University of Dusseldorf, Düsseldorf, Düsseldorf, Germany

${ }^{*}$ Corresponding author: M Li-Weber, Tumor Immunology Program D030, German Cancer Research Center (DKFZ), Im Neuenheimer Feld 280, Heidelberg 69120, Germany. Tel: + 496221 423748; Fax: + 496221 411715; E-mail: m.li-weber@dkfz-heidelberg.de

Keywords: anticancer drug; apoptosis; TRAIL; c-FLIP; ATL

Abbreviations: TNF, tumor necrosis factor; TRAIL, TNF-related apoptosis-inducing ligand; MAPK, mitogen-activated protein kinases; ERK, extracellular-signalregulated kinase

Received 13.1.10; revised 08.6.10; accepted 13.7.10; Edited by J Tschopp; published online 13.8.10
} 
C
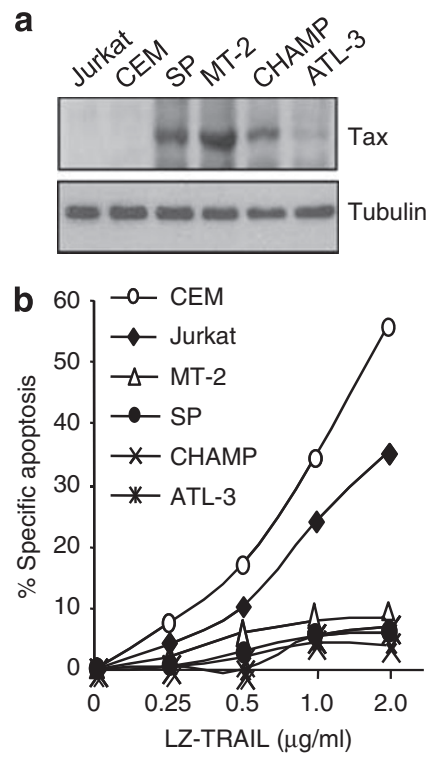
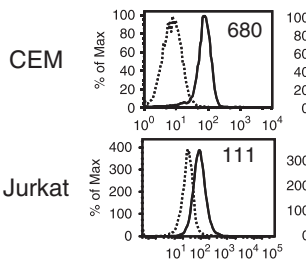

ATL-3
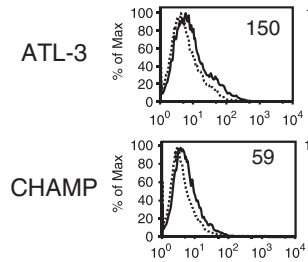

$\mathrm{SP}$

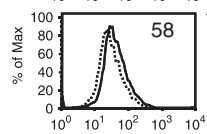

MT-2

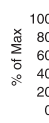

TRAIL-R2
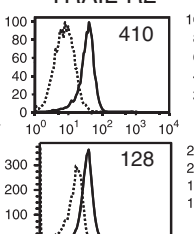

$10^{1} 10^{2} 10^{3} 10^{4} 10^{5}$
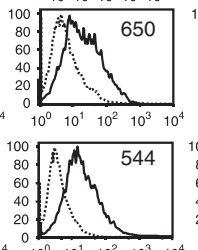

$10^{0} 10^{1} 10^{2} 10^{3} 10^{4}$
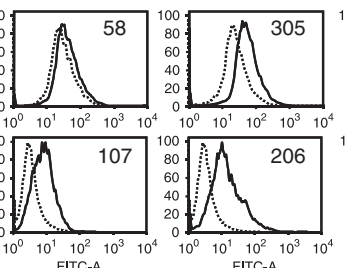

FITC-A

........ Isotype-matched control IgG1 mAb

- Expression levels of TRAIL receptors
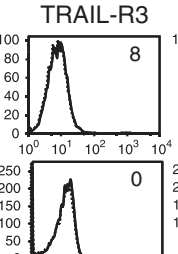

$10^{1} 10^{2} 10^{3} 10^{4} 10^{5}$
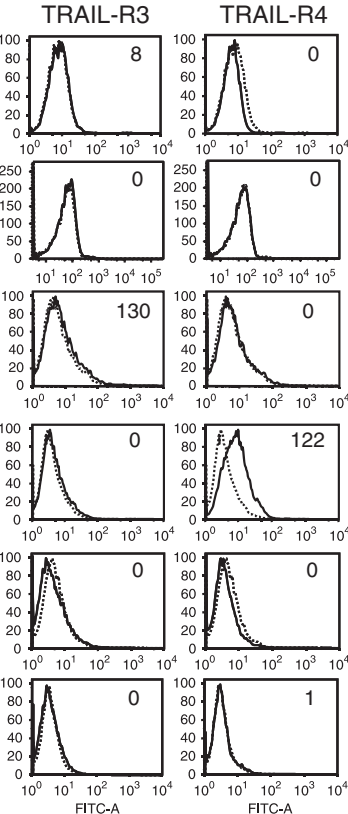$$
\text { FIT }
$$

(n)

Figure 1 HTLV-1-associated ATL cells are resistant to TRAIL and express higher levels of C-FLIP. (a) HTLV-1-associated ATL cell lines are characterized by the expression of the viral protein Tax. HTLV-1-infected ATL cell lines SP, MT-2, CHAMP and ATL-3 and non-infected cell lines Jurkat and CEM were subjected to western blot with an antibody against Tax. (b) HTLV-1-infected ATL cell lines are resistant to TRAIL-induced apoptosis. CEM, Jurkat and the HTLV-1-infected ATL cell lines were treated with different concentrations of TRAIL. Apoptotic cell death was determined by DNA fragmentation. The results are representative of two independent experiments. (c) All HTLV-1-infected ATL cell lines express TRAIL receptors 1 and 2. The numbers given in every graph are the MFI of the receptor staining after subtraction of MFI of isotype-matched control. The data are representative of two independent experiments. (d) All HTLV-1-infected cell lines express elevated c-FLIP proteins. Total cell lysates from HTLV-1-infected and non-infected cell lines were analyzed for c-FLIP expression by western blot

extracellular-signal-regulated kinase (ERK) and the phosphatidylinositol 3-kinase (PI3K) both have been shown to regulate c-FLIP expression. ${ }^{16-20}$ Furthermore, c-FLIP protein turnover has been shown to be actively regulated by ubiquitinmediated proteasomal degradation. ${ }^{21,22}$ Especially, the short form of $\mathrm{c}$-FLIP proteins, $\mathrm{c}-$ FLIP $_{\mathrm{S}}$, contains a unique $\mathrm{C}$ terminus that confers its preferred ubiquitination and, thus, a considerably shorter half-life than the long form of c-FLIP $\left(\mathrm{c}-\mathrm{FLIP}_{\mathrm{L}}\right)^{21}$ Activation of the stress-response $\mathrm{C}$-Jun $\mathrm{N}$-terminal protein kinase (JNK) may also promote C-FLIP $\mathrm{P}_{\mathrm{L}}$ ubiquitination through phosphorylation of the E3 ubiquitin ligase Itch. $^{22,23}$

We have previously shown that Rocaglamide (Roc), a naturally occurring herbal compound, derived from the traditional Chinese medicinal plant Aglaia (for reviews, see Proksch et al. ${ }^{24}$ and Kim et al. ${ }^{25}$ ), preferentially induces apoptosis in malignant but not normal proliferating lymphocytes. $^{26}$ This preference has been partially explained by its activation of the stress-response mitogen-activated protein kinases (MAPK) p38 and JNK in contrast to suppression of survival MAPK ERK activity in malignant, but not normal cells. ${ }^{26}$ Recently, in vitro and an in vivo mouse T-cell leukemia model, we showed high toxicity to tumor cells and only minor toxicity towards normal cells, e.g., activated $\mathrm{T}$ cells. ${ }^{26,27}$ Toxicity and cell death is mediated through suppression of c-FLIP expression at the transcriptional level by blocking NF-AT activation. ${ }^{27}$ Here, we further investigated whether Roc inhibits c-FLIP expression by additional mechanisms and whether it can be used as a sensitizer of receptor-mediated apoptosis. We show that Roc sensitizes resistant ATL cells to receptor (TRAIL-R1/2)-mediated apoptosis by translational suppression of c-FLIP through inactivation of the translation initiation factor 4E (elF4E). Our studies demonstrate that Roc may serve as an adjuvant of TRAIL-based therapies in c-FLIP-overexpressing malignant cells.

\section{Results}

Roc inhibits c-FLIP expression. Recently, we have shown that Roc suppresses activation-induced expression of c-FLIP in leukemic $T$ cells at the transcriptional level by downregulation of NF-AT activity. ${ }^{27}$ In that study, we noticed that the basal level of c-FLIP expression (without activation) was also reduced upon Roc treatment. Thus, we asked whether Roc can sensitize ATL cells to receptor-mediated apoptosis through suppression of c-FLIP expression. To investigate this question, we randomly chose four different ATL cell lines derived from HTLV-1-infected ATL patients: SP, MT-2, CHAMP and ATL-3. All cell lines are characterized by the expression of the viral transactivator protein Tax (Figure 1a), and are resistant to TRAIL-mediated apoptosis compared with non-HTLV-1infected leukemic cell lines, e.g., Jurkat and CEM (Figure 1b). All ATL cell lines express TRAIL-R1 and TRAIL-R2 at levels comparable with those of CEM and Jurkat cells (Figure 1c). Thus, resistance to TRAIL-induced 


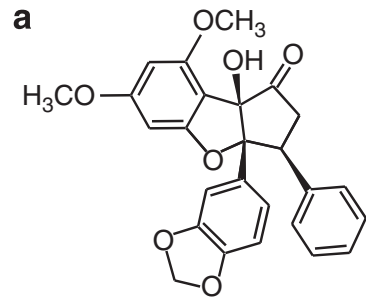

1-oxo-11,12-methylendioxyrocaglaol

b

C
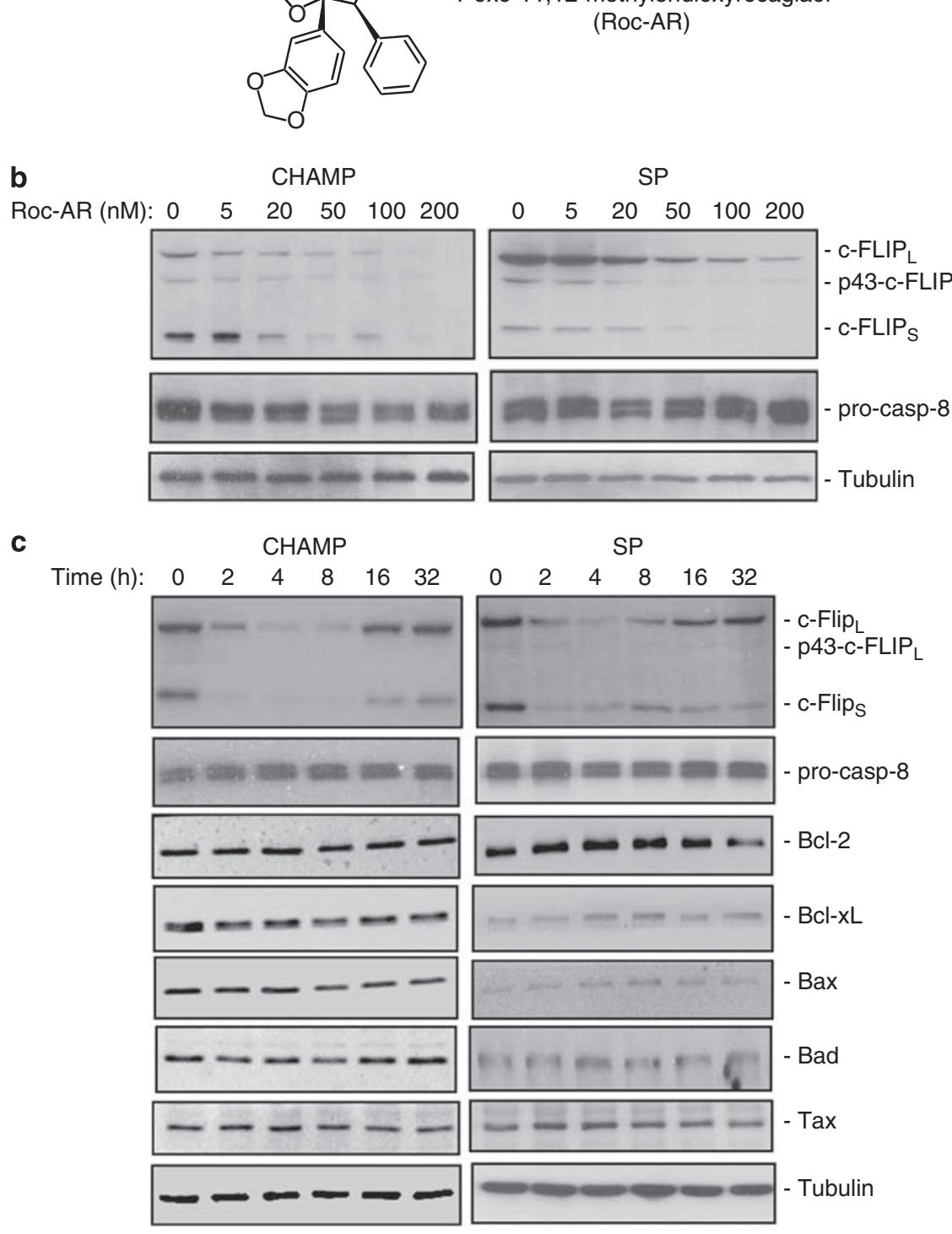
(Roc-AR)

Figure 2 Roc-AR inhibits C-FLIP expression in HTLV-1-associated ATL cells. (a) Chemical structure of Roc-AR. (b) Roc-AR inhibits c-FLIP expression in a dose-dependent manner. HTLV-1-associated ATL cell lines CHAMP and SP were treated with different concentrations of Roc-AR for $4 \mathrm{~h}$. c-FLIP protein expression levels were analyzed by western blot with $\alpha-c-F L I P$ antibody. Protein expression levels of procaspase- 8 and tubulin were used to control for equal protein loadings. The results are representative of two independent experiments. (c) Kinetic analysis Roc-AR-mediated inhibition of c-FLIP expression. CHAMP and SP cells were treated with $100 \mathrm{nM}$ Roc-AR for different times. The cell lysates were subjected to western blot analysis of expression levels of c-FLIP, procaspase-8, Bcl-2 family proteins, Tax and tubulin as indicated. The results are representative of at least three independent experiments

apoptosis in these cells is not due to a lack of sufficient amounts of cell surface TRAIL receptors. Consistent with previous studies, ${ }^{12,13}$ all Tax-expressing ATL cell lines express elevated levels of C-FLIP, Bcl-xL and $\mathrm{Bcl}-2$ proteins (Figure 1d). We then treated these cells with different concentrations of a Roc derivative, Roc-AR (Figure 2a). The experiment showed that Roc-AR inhibited c-FLIP expression in a dose- and time-dependent manner (Figure $2 \mathrm{~b}$ and $\mathrm{c}$, only CHAMP and SP are shown. Similar results were observed in the other two cell lines). In contrast, the expression levels of $\mathrm{Bcl}-2$ and $\mathrm{Bcl}-\mathrm{xL}$ were not downregulated by the concentration of Roc that suppresses c-FLIP expression (Figure 2c). Roc treatment also did not affect the expression levels of the Tax protein (Figure 2c). Downregulation of c-FLIP expression was also observed by another two Roc derivatives Roc-A and Roc-Q (data not shown). As controls, the expression levels of procaspase-8 and tubulin were unchanged.

Roc sensitizes CD95L- and TRAIL-mediated apoptosis in ATL, but not in normal T cells. As knocking-down of c-FLIP by an siRNA approach has been shown to sufficiently 

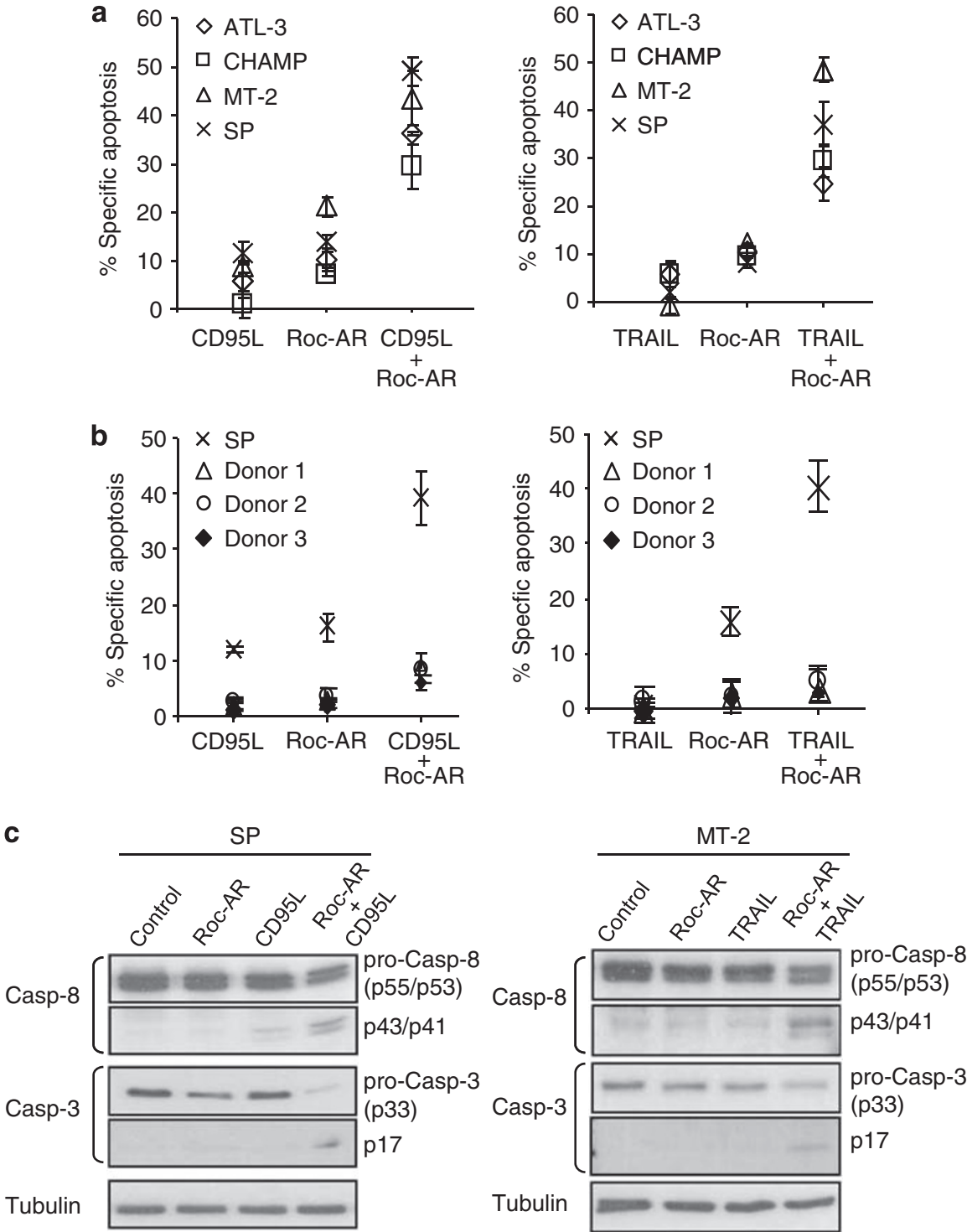

Figure 3 Roc-AR sensitizes HTLV-1-associated ATL but not healthy T cells towards CD95L- and TRAIL-induced apoptosis. (a) Roc-AR sensitizes HTLV-1-associated ATL cells towards CD95L- and TRAIL-induced apoptosis. The HTLV-1-associated ATL cell lines SP, MT-2, ATL-3 and CHAMP were untreated or treated either with Roc-AR $(100 \mathrm{nM})$, LZ-CD95L $(100 \mathrm{ng} / \mathrm{ml})$ or Superkiller-TRAIL $(100 \mathrm{ng} / \mathrm{ml})$ alone or in combination for $16 \mathrm{~h}$. Apoptotic cell death was determined by DNA fragmentation. The results shown are average of three independent experiments. (b) Roc-AR does not sensitize healthy T cells towards CD95L- and TRAIL-induced apoptosis. Freshly isolated peripheral blood T cells from three healthy donors were untreated or treated with Roc-AR, LZ-CD95L or Superkiller-TRAIL alone or in combination as in (a) for $16 \mathrm{~h}$. SP cells were used as a positive control. Apoptotic cell death was determined by DNA fragmentation. Results are average of three independent experiments. (c) Roc-AR enhances CD95L- and TRAIL-induced procaspase-8 processing. SP and MT-2 cells were untreated or treated with indicated drugs either alone or in combination as in (a) for $4 \mathrm{~h}$. Cell lysates were subjected to western blot analysis with antibodies against caspases-8 and 3 as indicated. Equal protein loading was controlled by tubulin

restore the sensibility of Tax-expressing leukemic cells to receptor-mediated apoptosis, ${ }^{12,13}$ we predicted that inhibition of c-FLIP expression by Roc would lead to sensitization of HTLV-1-infected ATL cells to receptor-mediated apoptosis. As expected, treatment of the ATL cells in the presence of Roc-AR synergistically increased CD95L- and TRAILinduced apoptotic cell death (Figure 3a). In contrast, RocAR did not sensitize normal peripheral blood $T$ cells to CD95L- and TRAIL-mediated apoptosis (Figure 3b). The ability of Roc-AR to enhance receptor-mediated apoptosis was further demonstrated by western blot showing that the activity of caspase-8, the main caspase involved in the extrinsic death pathway, was enhanced by the combination treatment that could be observed as early as $4 \mathrm{~h}$ after treatment (Figure $3 \mathrm{c}$ ). The sensitization is not due to enhanced expression of the receptors as Roc-AR did not influence cell surface expression levels of CD95 and TRAIL receptors after $12 \mathrm{~h}$ treatment (Supplementary Figure S1). These data demonstrate that Roc can sensitize HTLV-1infected leukemic cells towards CD95L- and TRAIL-mediated apoptosis by downregulation of c-FLIP.

Roc inhibits protein synthesis. We first investigated whether Roc-AR inhibits c-FLIP mRNA expression in 
a
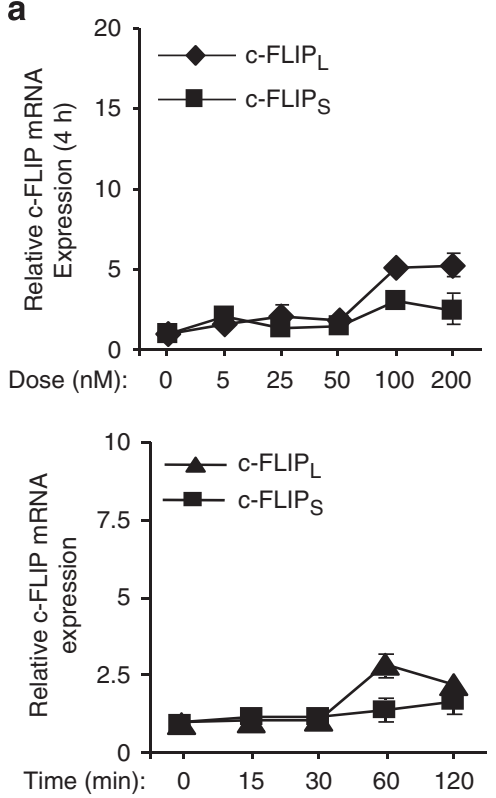

b
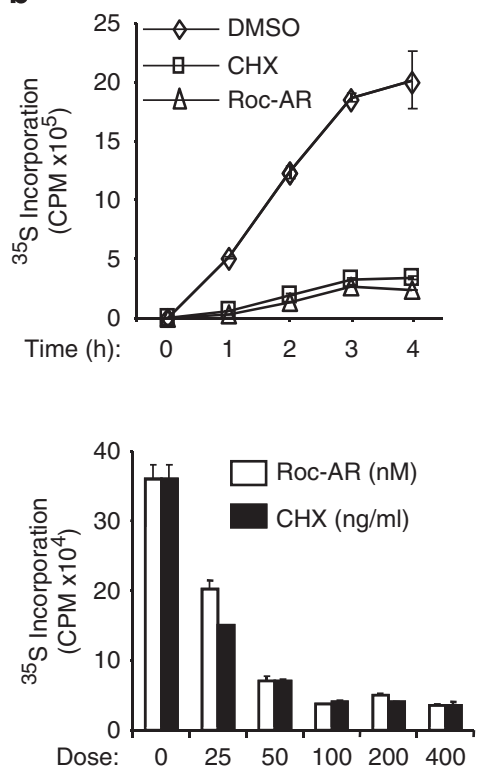
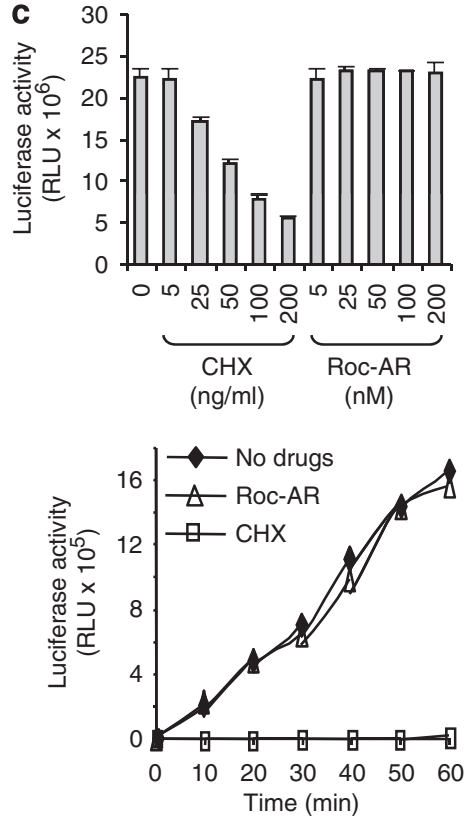

Figure 4 Roc-AR inhibits c-FLIP expression at the translational level. (a) Roc-AR does not inhibit c-FLIP mRNA expression. SP cells were treated with either different concentrations of Roc-AR (upper panel) or $100 \mathrm{nM}$ Roc-AR for different times (lower panel) as indicated. Total mRNA was isolated and mRNA expression levels of $c-F L I P_{L}$ and c-FLIPS were analyzed by quantitative real-time PCR. Results are average of three assays. (b) Roc-AR inhibits protein synthesis. SP cells were incubated with ${ }^{35}$ S-labelled methionine for $1 \mathrm{~h}$ and were then treated with either $100 \mathrm{nM}$ Roc-AR or $100 \mathrm{ng} / \mathrm{ml}$ of cycloheximide (CHX) for the indicated times (upper panel) or treated with different concentrations of Roc-AR for $1 \mathrm{~h}$ (lower panel). The incorporation of ${ }^{35} \mathrm{~S}$-methionine was monitored as described in Materials and Methods section. Data are representative of two experiments. (c) Roc-AR does not inhibit protein synthesis in vitro. The cell-free rabbit reticulocyte lysate in vitro translation assay was performed in the absence or presence of different concentrations of Roc-AR or CHX for $60 \mathrm{~min}$ (upper panel) or with $100 \mathrm{nM}$ Roc-AR or $100 \mathrm{ng} / \mathrm{ml}$ of CHX for the indicated times (lower panel). Firefly luciferase activity (RLU) was measured as described in Materials and Methods section. Data are representative of triplicate experiments

HTLV-1-infected ATL cells. SP cells were treated with different doses of Roc-AR for $4 \mathrm{~h}$ or with $100 \mathrm{nM}$ Roc-AR for different times. Quantitative real-time PCR analysis showed that Roc-AR treatment had no influence on c-FLIP mRNA expression levels (Figure 4a). This experiment indicates that Roc-mediated downregulation of c-FLIP in HTLV-1-infected ATL cells is not regulated at the transcriptional level.

Roc belongs to a group of cyclopenta[b]benzofuran derivatives. Several cyclopenta[b]benzofuran derivatives have been shown to inhibit protein synthesis without affecting DNA and RNA synthesis. ${ }^{25}$ Therefore, we carried out a ${ }^{35}$ S-methionine incorporation assay to examine whether Roc-AR downregulates c-FLIP expression through inhibition of protein synthesis. In parallel, cycloheximide $(\mathrm{CHX})$ was used as a control, which inhibits protein biosynthesis by the ribosomal machinery. ${ }^{28}$ The experiment showed that Roc-AR strongly inhibited ${ }^{35} \mathrm{~S}$-methionine incorporation with an $\mathrm{IC}_{50}$ of approximately $30 \mathrm{nM}$ (Figure $4 \mathrm{~b}$ ). These data suggest that Roc-AR may downregulate c-FLIP expression through inhibition of translation.

To further investigate whether Roc directly inhibits the protein synthesis machinery, we carried out an in vitro translation assay using a rabbit reticulocyte cell-free system. Luciferase mRNA was incubated with the rabbit reticulocyte lysate in the absence or presence of different concentrations of Roc-AR (0-200 nM) or CHX (0-200 ng/ml). Luciferase protein synthesis was quantified by luminescense.
The experiment showed that $\mathrm{CHX}$ blocked protein synthesis in a dose- and time-dependent manner (Figure 4c). In contrast, $100 \mathrm{nM}$ of Roc-AR, which was shown to completely block ${ }^{35} \mathrm{~S}$-methionine incorporation (Figure $4 \mathrm{~b}$ ), showed no effect on protein synthesis in the rabbit reticulocyte cell-free system (Figure 4c). These data indicate that Roc does not directly inhibit the translation machinery, but rather acts through inhibition of the signaling pathway that is required for translation.

Roc inhibits the MEK-ERK-MNK-elF4E signaling pathway. Initiation of translation involves a highly regulated process. The rate-limiting step of translation is the binding of the initiation factor elF4E to the mRNA $5^{\prime}$ cap structure. $^{29}$ The activity of elF4E is regulated by two major signaling pathways, the ERK and the PI3K pathway. ${ }^{29-31}$ ERK phosphorylates the kinase Mnk, which in turn phosphorylates elF4E. Phosphorylation of elF4E increases its affinity for the $5^{\prime}$ cap structure and thereby stimulates translation (schematically depicted in Figure 5a). PI3K activates eIF4E through activation of the kinase mammalian target of rapamycin (mTOR). ${ }^{29-31}$ mTOR activation disrupts the interaction between 4E-BP1 and elF4E, allowing elF4E to bind to the $5^{\prime}$ cap structure (schematically depicted in Figure $5 b$ ). Both, ERK and PI3K pathways have been shown to regulate c-FLIP expression. ${ }^{17-20}$ We have previously shown that Roc-A, a Roc derivative with a structure similar to Roc-AR, can downregulate ERK activity in different 
a

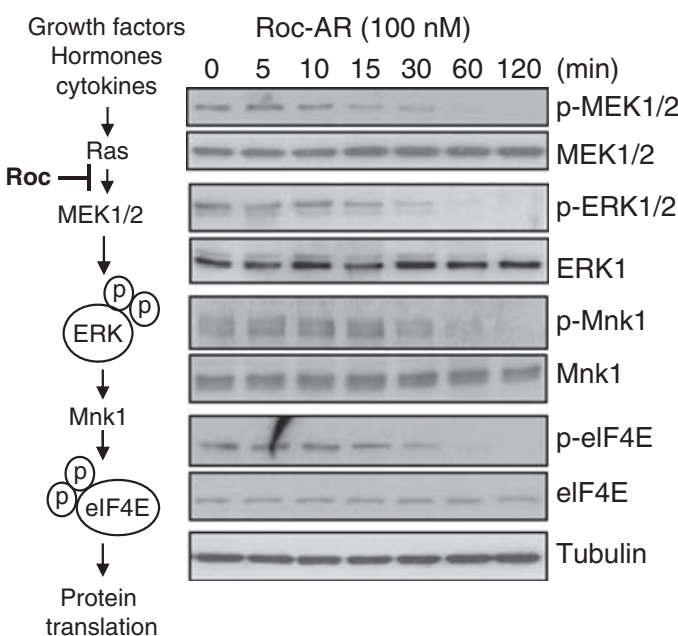

C b

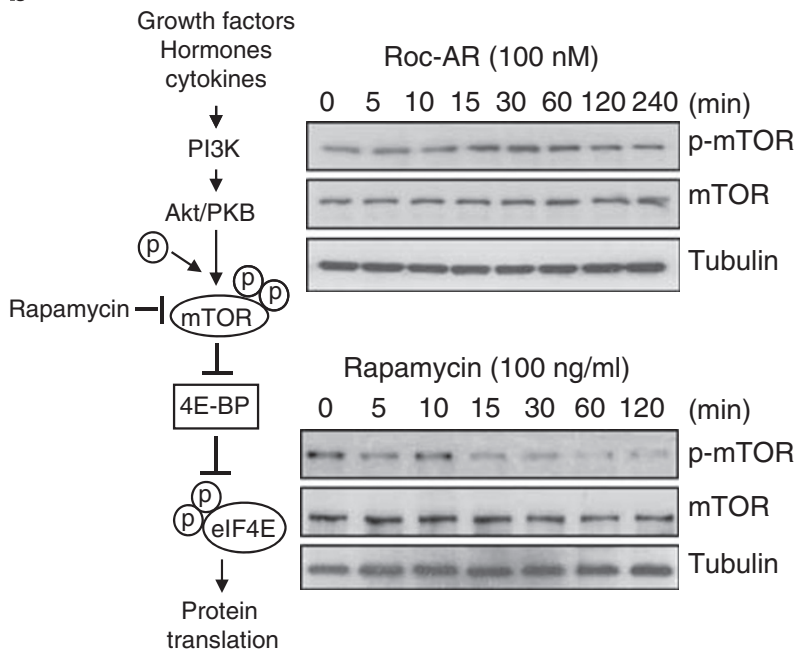

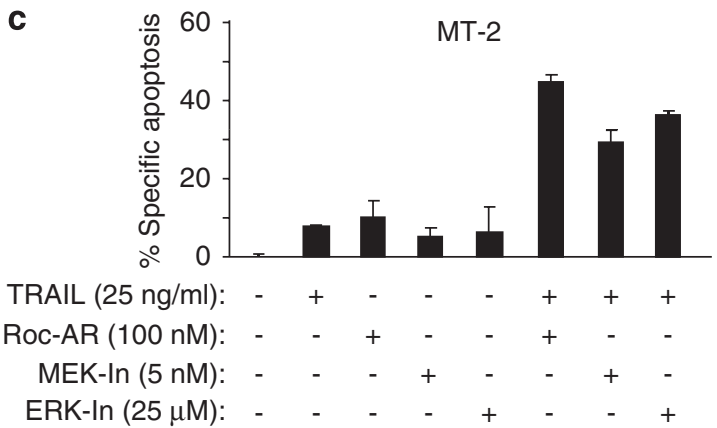

Figure 5 Roc-AR blocks the MEK-ERK-Mnk-elF4E, but not the mTOR pathway. (a) Roc-AR blocks the MEK-ERK-Mnk-elF4E pathway. SP cells were treated with $100 \mathrm{nM}$ Roc-AR for 0-120 min. Total cell lysates were subjected to western blot by the indicated antibodies. (b) Roc-AR does not affect the mTOR pathway. SP cells were treated with either $100 \mathrm{nM}$ Roc-AR (upper panel) or $100 \mathrm{ng} / \mathrm{ml}$ of Rapamycin (lower panel) as a positive control for the indicated times. Total cell lysates were analyzed by western blot with the indicated antibodies. Data are representative of two reproducible experiments. (c) MEK and ERK inhibitors sensitize HTLV-1-associated ATL cells towards TRAIL-induced apoptosis. MT-2 cells were untreated or treated with Roc-AR or specific inhibitors of MEK (MEK-In) or ERK (ERK-In) in the presence or absence of Superkiller-TRAIL for $24 \mathrm{~h}$. Apoptotic cell death was determined by DNA fragmentation. The results shown are representative of two independent experiments each in triplicates

leukemic cell lines and in leukemic samples from patients. ${ }^{26}$ To investigate whether Roc-AR could also inhibit the ERK pathway in HTLV-1-infected ATL cells, SP cells were treated with Roc-AR (100 nM) for different times, and the phosphorylation status of MEK1/2, ERK, MNK1 and elF4E was analyzed by western blot. The experiments showed that Roc-AR inhibits the phosphorylation of $M E K 1 / 2$, ERK, MNK and elF4E and the inhibition could be detected as early as $30 \mathrm{~min}$ after Roc-AR treatment (Figure 5a). We then investigated whether Roc-AR also interferes with the PI3K-mTOR pathway. The experiment showed that Roc-AR at a dose that inhibits c-FLIP protein expression did not inhibit mTOR phosphorylation (Figure 5b). As a positive control, phosphorylation of mTOR was blocked by Rapamycin in a time-dependent manner (Figure 5b). Therefore, Roc can inhibit protein synthesis, e.g., of c-FLIP by blocking the MEK-ERK-MNK-elF4E signaling pathway.

To further investigate the role of the MEK-ERK-MNK signaling pathway in regulation of the receptor-mediated apoptosis, we carried out an experiment using specific inhibitors that target activities of MEK and ERK, respectively.
As expected, inhibition of MEK or ERK alone could sensitize TRAIL-induced apoptosis (Figure 5c). Thus, Roc-mediated inhibition of the MEK-ERK signaling pathway contributes to sensitization of TRAIL-induced apoptosis in HTLV-associated ATL cells.

It has been shown that JNK can promote C-FLIP ubiquitination and degradation through phosphorylation of the E3 ubiquitin ligase Itch. ${ }^{22}$ We have previously shown that JNK can be transiently activated by Roc. ${ }^{26,27}$ Thus, Roc-mediated downregulation of C-FLIP $\mathrm{P}_{\mathrm{L}}$ might also involve a JNK-mediated mechanism. To investigate this possibility, we carried out the experiment in the presence of the JNK-specific inhibitor SP600125. However, we did not see clear reversal of the Roc effect using different concentrations of SP600125 (Supplementary Figure S2). This unresponsiveness to the JNK inhibitor may be explained by the fact that (1) turnover of $\mathrm{C}-$ FLIP $_{\mathrm{L}}$ but not $\mathrm{C}-$ FLIP $_{\mathrm{S}}$ is controlled by JNK activity. ${ }^{22}$ Therefore, preventing degradation of $\mathrm{C}-$ FLIP $_{\mathrm{L}}$ alone may not be sufficient to overcome Roc-mediated sensitization. (2) JNK is only transiently activated by Roc $(0.5-2 \mathrm{~h}){ }^{26}$ Thus, such short activation may not have a main role in degradation of C-FLIP $P_{L}$. (3) As Roc inhibits de novo protein synthesis, 


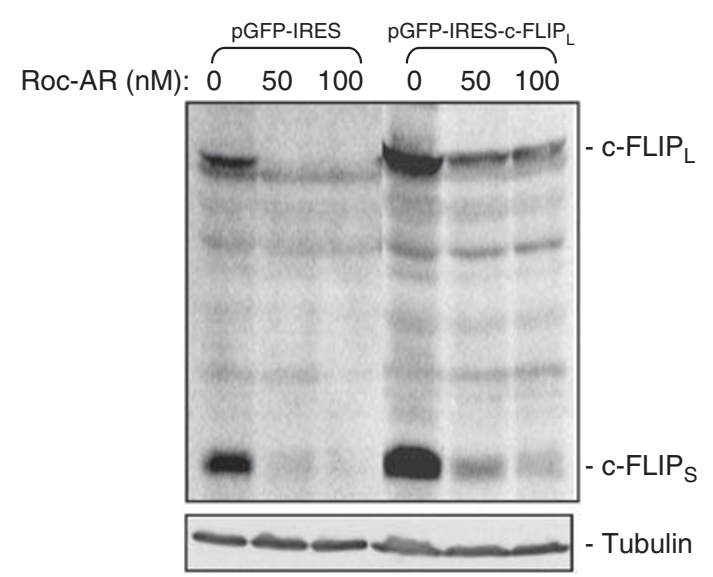

Figure 6 Roc does not inhibit IRES-mediated expression of C-FLIP. HeLa cells were transfected with a pGFP-IRES-c-FLIP ${ }_{L}$ expression plasmid and its parental empty plasmid pGFP-IRES. After $24 \mathrm{~h}$ transfection, cells were split and treated without or with different doses of Roc-AR for $8 \mathrm{~h}$. The expression levels of c-FLIP proteins were analyzed by western blot. The result is representative of two independent transfection studies

the $\mathrm{C}-\mathrm{FLIP}_{\mathrm{L}}$ level will be eventually reduced even in the presence of the JNK inhibitor.

To further confirm that downregulation of c-FLIP expression by $\mathrm{Roc}$ is regulated through inhibition of cap-dependent translation, we examined the effect of Roc on the expression of c-FLIP whose translation is regulated through IRES, a translation mechanism independent of cap-structure. ${ }^{32}$ The experiments showed that Roc treatment resulted in the inhibition of the endogenous $C-F L I P_{L}$ and $C-F L I P S$ protein expression in HeLa cells (Figure 6, left panel, transfected with the empty plasmid pGFP-IRES). In contrast, Roc failed to inhibit $c-F_{L} P_{L}$ ectopically expressed by the pGFP-IRES-cFLIP $_{L}$ plasmid but could still inhibit the endogenous C-FLIP and $\mathrm{C}-\mathrm{FLIP}_{\mathrm{S}}$ expression (Figure 6, right panel, transfected with pGFP-IRES-c-FLIP ). This study further demonstrates that Roc inhibits translation by targeting the elF4E-mediated translation.

\section{Discussion}

Although TRAIL is a promising anticancer agent that kills various cancer cells with no toxicity to normal cells, many cancer cells remain resistant to TRAIL. For instance, most leukemias including HTLV-1-associated ATL show restricted sensitivity to TRAIL. ${ }^{7}$ We and others have recently shown that HTLV-1 Tax, the major HTLV-1 protein with oncogenic potential, confers resistance to receptor-mediated apoptosis by enhancing c-FLIP expression. ${ }^{12,13}$ This underscores the demand of therapies with agents that can target c-FLIP expression to enhance the TRAIL-signalling pathway. In this study, we show that Roc-AR enhances TRAIL-mediated apoptosis in HTLV-1-infected ATL cells by downregulation of c-FLIP expression.

Two main signaling pathways, the Ras-ERK and the PI3K pathway, have been implicated in controlling C-FLIP expression. ${ }^{16-20}$ The Ras-ERK pathway is also the target of the HTLV-1 oncogenic protein Tax. ${ }^{33,34}$ Recently, Ras-ERK signaling was shown to be particularly important for Taxmediated protection of cells from apoptosis. ${ }^{35}$ Our study demonstrates that Roc can block the Ras-ERK signaling pathway and thereby downregulate c-FLIP expression through inhibition of elF4E function, a key factor that controls the rate-limiting step of translation. ${ }^{29}$

Although inhibition of elF4E function will lead to inhibition of translation in general, only the expression levels of c-FLIP proteins were shown to be downregulated by Roc treatment. No suppression of the expression levels of $\mathrm{Bcl}-2, \mathrm{Bcl}-\mathrm{xL}$ and Tax was seen in Roc-treated ATL cells. It is known that c-FLIP turnover is actively regulated by a mechanism of ubiquitinmediated proteasomal degradation and, therefore, c-FLIP has a short half-life. ${ }^{21,22}$ Especially, c-FLIPS protein contains a unique $\mathrm{C}$ terminus that confers its preferred ubiquitinilation and, thus, has an even shorter half-life than the $\mathrm{C}$-FLIP ${ }_{\mathrm{L}}{ }^{21}$ Thus, inhibition of de novo protein synthesis by Roc leads to downregulation of only short-lived proteins, such as c-FLIP, especially c-FLIP . c-FLIP expression has also been shown to be controlled by the PI3K pathway. ${ }^{19,20}$ However, in our experiments, Roc did not show an inhibitory effect on the PI3K pathway.

So far, HTLV-1-associated ATL is incurable by the presently known therapies. ${ }^{9}$ As TRAIL is a promising anticancer agent, this study is aimed to explore an approach to overcome TRAIL-resistance in HTLV-1-associated ATL cells. Sensitivity and resistance of HTLV-1-associated ATL cells to other chemotherapeutic drugs are another important issue. As Roc inhibits the MEK-ERK signaling pathway, we speculate that combinations of Roc with other chemotherapeutic drugs that target the signaling pathways different from Roc could have beneficial effects on the treatment. Future studies are planned to investigate this issue.

In summary, we demonstrate that Roc inhibits expression of c-FLIP at the translational level, and thereby breaks TRAIL resistance in HTLV-1-infected ATL cells. We also show that Roc does not directly inhibit the translation machinery, instead Roc inhibits the ERK pathway leading to inhibition of phosphorylation of elF4E, a key factor for controlling the rate-limiting step of translation. So far, therapy shows only very limited benefit to HTLV-1-associated ATL patients because of resistance of the tumor cells to apoptosis. Our study raises the possibility to develop Roc as a TRAIL adjuvant for treatment of HTLV-1-associated ATL. As many tumors express elevated levels of C-FLIP and siRNA, knockdown of c-FLIP has been shown to sensitize these cells to death ligands; Roc may also be used to enhance the success of TRAIL therapy in c-FLIP-overexpressing cancers.

\section{Materials and Methods}

Cells and cell cultures. The human malignant cell lines used in this study are the human leukemic T-cell lines CEM and Jurkat (J16) and the HTLV-1 ATL-derived cell lines SP, MT-2, CHAMP and ATL-3. Human peripheral blood T cells were prepared as described previously ${ }^{27}$ and were more than $90 \% \mathrm{CD}^{+}$. All cells were cultured in RPMI 1640 medium (GIBCO Laboratories, Grand Island, NY, USA) supplemented with $10 \% \mathrm{FCS}, 50 \mu \mathrm{g} / \mathrm{ml}$ gentamicin (GIBCO Laboratories), $6 \mathrm{mM}$ HEPES (GIBCO Laboratories, $1 \mathrm{M}$ solution) and $2 \mathrm{mM}$ L-glutamine (GIBCO Laboratories, $200 \mathrm{mM}$ solution) at $37^{\circ} \mathrm{C}$ and $5 \% \mathrm{CO}_{2}$.

Determination of apoptosis. Cells were plated in triplicates and treated for the indicated periods of time at $37^{\circ} \mathrm{C}$ with different doses of Roc-AR (>98\% pure, 
assessed by HPLC), Superkiller-TRAIL (Alexis Biochemicals, San Diego, CA, USA) or LZ-CD95 $\mathrm{L}^{5}$ alone, or in combinations as indicated in the respective figures. Apoptotic cell death was determined by analysis of DNA fragmentation (\% DNA fragmentation) as previously described. ${ }^{12}$ Specific apoptosis was calculated as (percentage of experimental apoptosis-percentage of spontaneous apoptosis)/ (100-percentage of spontaneous apoptosis) $\times 100$. Inhibitors used to block the ERK pathway are ERK-specific inhibitor 3-(2-Aminoethyl)-5-((4-ethoxyphenyl)methylene)2,4-thiazolidinedione $\mathrm{HCl}$ purchased from Calbiochem (Cat. no. 328006) and the MEK1/2 inhibitor III (PD0325901) purchased from MERCK (Calbiochem, Darmstadt, Germany).

Western blot analysis. For each sample, $1 \times 10^{7}$ cells were lysed as described previously. ${ }^{26}$ Equal amounts of proteins were separated on $7.5-13 \%$ SDSPAGE depending on the molecular sizes of the proteins, blotted onto a nitrocellulose membrane (Amersham Biosciences, Little Chalfon, UK) and blocked with 5\% non-fat dry milk in PBS/Tween (0.05\% Tween-20 in PBS). The following antibodies were used: anti-caspase-3 polyclonal antibody (Cell Signaling), anti-ERK1 and antiphospho-ERK (Cell Signaling, Danvers, MA, USA), anti-MEK and anti-phospho MEK antibody (Cell Signaling), anti-mTOR (2972) and anti-phospho-mTOR (2971) (Cell Signaling), anti-Mnk1 (C-20) (Santa Cruz Biotechnology, Heidelberg, Germany), antiphospho-Mnk1 (2111) (Cell Signaling), anti-elF4E (9742) and anti-phospho-elF4E (9742) (Cell Signaling), anti-actin, anti-tubulin (Sigma-Aldrich, St. Louis, MO, USA), anti-TRAIL-R1-R4 (Alexis Biochemicals) and anti-Tax (hybridomas, clone 168B17-4634, NIH AIDS Research, NIH, Rockville, MD, USA). The caspase-8 mAb C15 (mouse IgG2b) recognizes the p18 subunit of caspase-8, the c-FLIP mAb NF6, the agonistic anti-CD95 mAb were generated in our laboratory as described previously. ${ }^{27}$ For stripping, blots were incubated for $30 \mathrm{~min}$ in a buffer containing $62.5 \mathrm{mM} \mathrm{Tris} / \mathrm{HCl}, \mathrm{pH}$ $6.8,2 \%$ SDS and $100 \mathrm{mM} \beta$-mercaptoethanol at $56^{\circ} \mathrm{C}$. The blots were washed six times for $10 \mathrm{~min}$ in PBS/Tween and blocked again in $5 \%$ non-fat dry milk.

RNA isolation and quantitative real-time PCR. Extraction of total cellular RNA was performed using the NucleoSpin RNAll (Macherey-Nagel, Düren, Germany) extraction kit with $1 \times 10^{7}$ cells per preparation according to the manufacturer's instructions. One $\mu \mathrm{g}$ of RNA was reverse transcribed into cDNA with MuLV reverse transcriptase (Roche, Mannheim, Germany) in the presence of $50 \mu \mathrm{M}$ oligo-d(T) and $200 \mathrm{nM}$ dNTPs at $42^{\circ} \mathrm{C}$ for $45 \mathrm{~min}$. Aliquots $(1 / 4)$ of the resulting CDNA were then used as templates for PCR. The primers and conditions used for quantitative real-time PCR for C-FLIP,$C$-FLIP and $\beta$-actin (used as an internal standard) were described previously. ${ }^{12}$

In vivo protein synthesis assays. Protein synthesis was estimated by measuring the amount of incorporated ${ }^{35} \mathrm{~S}$-methionine. Briefly, cells $\left(1 \times 10^{6}\right)$ were incubated for $2 \mathrm{~h}$ with $1 \mathrm{ml}$ of methionine-free medium in a 24-well plate. Then $7 \mu \mathrm{Ci}$ pro well of ${ }^{35}$ S protein-labeling mix (PerkinElmer, Waltham, MA, USA) was added and cells were treated with different drugs as indicated. After incubation, cells were washed twice with PBS and lysed in ice-cold RIPA-lysis buffer for $15 \mathrm{~min}$ on ice and centrifuged (20 min, 13000 r.p.m.). Four $\mu$ l of each lysate was loaded on a filter paper, followed by precipitation with $10 \%$ trichloroacetic acid (TCA) by incubating the filter paper three times in TCA precipitation buffer for $5 \mathrm{~min}$. After drying, the filter papers were incubated in $1 \mathrm{ml}$ of Liquid Scintillation Cocktail solution (Beckman coulter, Brea, CA, USA) and radioactivity was determined with Liquid Scintillation counting.

In vitro translation assays. For the in vitro translation assay, the rabbit reticulocyte lysate system was used according to the manufacturer's instructions (Promega Corp., Mannheim, Germany). Briefly, $35 \mu \mathrm{l}$ cell-free rabbit reticulocyte lysate was incubated with $1 \mu \mathrm{g}$ luciferase mRNA at $30^{\circ} \mathrm{C}$ in the absence or presence of different concentrations of Roc-AR $(0-200 \mathrm{nM})$ or CHX $(0-200 \mathrm{ng} / \mathrm{ml})$ for the indicated times. Synthesis of luciferase protein was quantified by luciferase activity with a luminometer (Duolumat LB9507, Berthold, Wildbach, Germany).

Cell surface staining receptors. For analysis of surface expression levels of CD95 and TRAIL receptors, cells $\left(5 \times 10^{5}\right)$ were washed with PBS and incubated with $1 \mu \mathrm{g} / \mathrm{ml}$ of corresponding antibodies for $30 \mathrm{~min}$ at $4^{\circ} \mathrm{C}$, washed with PBS and incubated for $30 \mathrm{~min}$ with phycoerythrin-conjugated goat anti-mouse antibody (Dianova, Duabiva, Hamburg, Germany), and analyzed by flow cytometry with a FACScan Cytometer (Becton Dickinson, Heidelberg, Germany). The following antiTRAIL receptor antibodies were used: HS101 (TRAIL-R1), HS201 (TRAIL-R2), HS301 (Dc-R1) and HS402 (Dc-R2) (Alexis). The CD95 antibody is anti-APO-1 produced by our laboratory. ${ }^{27}$
Plasmid and transfection. The GFP-IRES-C-FLIPL plasmid expressing the $55 \mathrm{kDa}$ FLIP $\mathrm{L}$ protein under the control of IRES was constructed previously. ${ }^{36}$ Transient transfection was carried out with the polyethylenimine (PEI, MW 25000 ) (Sigma, Cat.: 40.872-7) based method. Briefly, plasmid DNA was suspended in Opti-MEM medium (GIBCO laboratories, Grand Island, NY, USA; Cat.: 51985) in a final concentration of $10 \mu \mathrm{g} / \mathrm{ml}$, and PEI was suspended in Opti-MEM in a final concentration of $20 \mu \mathrm{g} / \mathrm{ml}$. Before transfection, the plasmid and PEI solution were mixed by a $1: 1$ volume ratio and incubated at RT for $20 \mathrm{~min}$ to allow DNA/PEI complex formation. Three $\mathrm{ml}$ of DNA/PEI complex were drop-wise added into $10 \mathrm{~cm}$ cell culture dish with $50-70 \%$ confluence of growth. Cells were then cultured at $37^{\circ} \mathrm{C}$ until harvest.

\section{Conflict of interest}

The authors declare no conflict of interest.

Acknowledgements. This work was supported by the Israeli (MOST)-DKFZ Cooperation Project in Cancer Research. We thank Fricker $\mathrm{N}$ and Lavrik IN for providing the IRES-c-FLIP plasmid.

1. Debatin KM, Krammer PH. Death receptors in chemotherapy and cancer. Oncogene 2004; 23: 2950-2966.

2. Galluzzi L, Larochette N, Zamzami N, Kroemer G. Mitochondria as therapeutic targets for cancer chemotherapy. Oncogene 2006; 25: 4812-4830.

3. Johnstone RW, Frew AJ, Smyth MJ. The TRAIL apoptotic pathway in cancer onset, progression and therapy. Nat Rev Cancer 2008; 8: 782-798.

4. Krammer PH, Arnold R, Lavrik I. Life and death in peripheral T cells. Nat Rev Immunol 2007; 7: 532-542.

5. Walczak H, Miller RE, Ariail K, Gliniak B, Griffith TS, Kubin M et al. Tumoricidal activity of tumor necrosis factor-related apoptosis-inducing ligand in vivo. Nat Med 1999; 5 : $157-163$

6. Lawrence D, Shahrokh Z, Marsters S, Achilles K, Shih D, Mounho B et al. Differential hepatocyte toxicity of recombinant Apo2L/TRAIL versions. Nat Med 2001; 7: 383-385.

7. Wuchter C, Krappmann D, Cai Z, Ruppert V, Scheidereit C, Dörken B et al. In vitro susceptibility to TRAIL-induced apoptosis of acute leukemia cells in the context of TRAIL receptor gene expression and constitutive NF-kappa B activity. Leukemia 2001; 15: 921-928.

8. Ehrhardt H, Fulda S, Schmid I, Hiscott J, Debatin KM, Jeremias I. TRAIL induced survival and proliferation in cancer cells resistant towards TRAIL-induced apoptosis mediated by NF-kappaB. Oncogene 2003; 22: 3842-3852.

9. Matsuoka M, Jeang K. Human T-cell leukaemia virus type 1 (HTLV-1) infectivity and cellular transformation. Nat Rev Cancer 2007; 7: 270-280.

10. Hasegawa H, Yamada Y, Harasawa H, Tsuji T, Murata K, Sugahara K et al. Sensitivity of adult T-cell leukaemia lymphoma cells to tumour necrosis factor-related apoptosis-inducing ligand. Br J Haematol 2005; 128: 253-265.

11. Matsuda T, Almasan A, Tomita M, Uchihara JN, Masuda M, Ohshiro K et al. Resistance to Apo2 ligand (Apo2L)/tumor necrosis factor-related apoptosis-inducing ligand (TRAIL)mediated apoptosis and constitutive expression of Apo2L/TRAIL in human T-cell leukemia virus type 1-infected T-cell lines. J Virol 2005; 79: 1367-1378.

12. Krueger A, Fas SC, Giaisi M, Bleumink M, Merling A, Stumpf $C$ et al. HTLV-1 Tax protects against CD95-mediated apoptosis by induction of the cellular FLICE-inhibitory protein (c-FLIP). Blood 2006; 107: 3933-3939.

13. Okamoto K, Fujisawa J, Reth M, Yonehara S. Human T-cell leukemia virus type-I oncoprotein Tax inhibits Fas-mediated apoptosis by inducing cellular FLIP through activation of NF-kappaB. Genes Cells 2006; 11: 177-191.

14. Ueffing N, Schuster M, Keil E, Schulze-Osthoff K, Schmitz I. Up-regulation of c-FLIP short by NFAT contributes to apoptosis resistance of short-term activated T cells. Blood 2008; 112: $690-698$.

15. Micheau O, Lens S, Gaide O, Alevizopoulos K, Tschopp J. NF-kappaB signals induce the expression of C-FLIP. Mol Cell Biol 2001; 21: 5299-5305.

16. Yeh JH, Hsu SC, Han SH, Lai MZ. Mitogen-activated protein kinase kinase antagonized fas-associated death domain protein-mediated apoptosis by induced FLICE-inhibitory protein expression. J Exp Med 1998; 188: 1795-1802.

17. Aoudjit $F$, Vuori K. Matrix attachment regulates Fas-induced apoptosis in endothelial cells: a role for c-flip and implications for anoikis. J Cell Biol 2001; 152: 633-643.

18. Wang W, Prince CZ, Mou Y, Pollman MJ. Notch3 signaling in vascular smooth muscle cells induces c-FLIP expression via ERK/MAPK activation. Resistance to Fas ligand-induced apoptosis. J Biol Chem 2002; 277: 21723-21729.

19. Panka DJ, Mano T, Suhara T, Walsh K, Mier JW. Phosphatidylinositol 3-kinase/Akt activity regulates C-FLIP expression in tumor cells. J Biol Chem 2001; 276: 6893-6896. 
20. Nam SY, Jung GA, Hur GC, Chung HY, Kim WH, Seol DW et al. Upregulation of FLIP(S) by Akt, a possible inhibition mechanism of TRAlL-induced apoptosis in human gastric cancers. Cancer Sci 2003; 94: 1066-1073.

21. Poukkula M, Kaunisto A, Hietakangas V, Denessiouk K, Katajamäki T, Johnson MS et al. Rapid turnover of c-FLIPshort is determined by its unique C-terminal tail. J Biol Chem 2005 280: 27345-27355.

22. Chang L, Kamata H, Solinas G, Luo JL, Maeda S, Venuprasad K et al. The E3 ubiquitin ligase itch couples JNK activation to TNFalpha-induced cell death by inducing c-FLIP(L) turnover. Cell 2006; 124: 601-613.

23. Gao M, Karin M. Regulating the regulators: control of protein ubiquitination and ubiquitinlike modifications by extracellular stimuli. Mol Cell 2005; 19: 581-593.

24. Proksch P, Edrada RA, Ebel R, Bohnenstengel FI, Nugroho BW. Chemistry and biological activity of rocaglamide derivatives and related compounds in Aglaia species (Meliaceae). Cur Org Chem 2001; 5: 923-938.

25. Kim S, Salim AA, Swanson SM, Kinghorn AD. Potential of cyclopenta[b]benzofurans from Aglaia species in cancer chemotherapy. Anticancer Agents Med Chem 2006; 6: 319-345

26. Zhu JY, Lavrik IN, Mahlknecht U, Giaisi M, Proksch P, Krammer PH et al. The tradition Chinese herbal compound rocaglamide preferentially induces apoptosis in leukemia cells by modulation of MAPK activities. Int J Cancer 2007; 121: 839-1846.

27. Zhu JY, Giaisi M, Köhler R, Müller WW, Mühleisen A, Proksch $P$ et al. Rocaglamide sensitizes leukemic $T$ cells to activation-induced cell death by differential regulation of CD95L and c-FLIP expression. Cell Death Differ 2009; 16: 1289-1299.
28. Lin SY, Mosteller RD, Hardesty B. The mechanism of sodium fluoride and cycloheximide inhibition of hemoglobin biosynthesis in the cell-free reticulocyte system. J Mol Biol 1966 21: $51-69$.

29. Gingras AC, Raught B, Sonenberg N. elF4 initiation factors: effectors of mRNA recruitment to ribosomes and regulators of translation. Annu Rev Biochem 1999; 68 913-963.

30. Kleijn M, Scheper GC, Voorma HO, Thomas AA. Regulation of translation initiation factors by signal transduction. Eur J Biochem 1998; 253: 531-544.

31. Wang X, Proud CG. The mTOR pathway in the control of protein synthesis. Physiology (Bethesda) 2006; 21: 362-369.

32. Jackson RJ, Hellen CU, Pestova TV. The mechanism of eukaryotic translation initiation and principles of its regulation. Nat Rev Mol Cell Biol 2010; 11: 113-127.

33. Wu K, Bottazzi ME, de la Fuente C, Deng L, Gitlin SD, Maddukuri A et al. Protein profile of tax-associated complexes. J Biol Chem 2004; 279: 495-508.

34. Pozzatti R, Vogel J, Jay G. The human T-lymphotropic virus type I tax gene can cooperate with the ras oncogene to induce neoplastic transformation of cells. Mol Cell Biol 1990; 10 413-417.

35. Vajente N, Trevisan R, Saggioro D. HTLV-1 Tax protein cooperates with Ras in protecting cells from apoptosis. Apoptosis 2009; 14: 153-163.

36. Fricker N, Beaudouin J, Richter P, Eils R, Krammer PH, Inna NL. Model based dissection of CD95 signaling dynamics reveals both a pro- and anti-apoptotic role of c-FLIPL. J Cell Biol (in press).

Supplementary Information accompanies the paper on Cell Death and Differentiation website (http://www.nature.com/cdd) 